\title{
Research of Cable Tunnel structure Settlement sensor based on FBG Technology
}

\author{
Huaguang Wang ${ }^{1}$, Mu Fang ${ }^{2}$; Nan Sun ${ }^{3}$ \\ ${ }^{1}$ State Grid Shandong Electric Power Company Maintenance Department, Shandong, 250001 \\ ${ }^{2}$ State Grid Shandong Electric Power Company Maintenance Department, Shandong, 250001 \\ ${ }^{3}$ Bandweaver Technologies Co., Ltd, Shanghai, 200000 \\ 43967125@163.com
}

\section{Keywords: FGB; Settlement Sensor; Tunnel Structure}

\begin{abstract}
With the rapid development of science and technology, sustained economic growth, social progress, a variety of engineering structures of today's society undergoing significant changes, material, structure, technology and other complex, and we will continue to large, complex , automation, and so the long-term direction. In China, large-scale dams, bridges, highways, high-speed railway, etc. large-scale projects are constantly under construction, for the effective monitoring and early warning of such large and complex engineering structure is very important and urgent. Optical fiber sensing technology has its unique advantages in the sensing field, anti-electromagnetic interference, corrosion resistance, insulation resistance, high sensitivity, passive and may constitute a large sensor network, after 30 years of research, optical fiber sensing technology continues to mature development and will play an important role in the field of geology health and safety monitoring.
\end{abstract}

\section{Introduction}

With China's rapid economic development, the government accelerated the pace of infrastructure construction, but also increased the funding this aspect, highway, railway, subway, bridges, tunnels and so on a large number of infrastructure construction. Therefore, the safety and health monitoring of these facilities is also very important for sensor technology and systems made more demands. Fiber optic sensor is used to detect various physical parameters of the project based on the unique properties of fiber optics and sensor characteristics. Fiber optic sensor compared to the traditional method has higher sensitivity, less susceptible to electromagnetic interference, good insulation properties, set the physical parameters of information acquisition and transmission in one, and a simple structure, small size, light weight, low power consumption and many other advantages, in the civil Major areas of engineering, aerospace, communications, electric power, petrochemical, intelligent security and health monitoring and so on geological research has an extremely wide significance and application prospects. FBG sensors to change the principle of Bragg reflection center wavelength is achieved by external parameters, the same with all the advantages of fiber optic sensors and associated properties. Press cycle and the refractive index of the Spatial classification, fiber grating can be divided evenly period fiber Bragg grating, Taper type grating, grating Moore, linearly chirped fiber grating, long period fiber grating (LPG) and shine / tilted fiber grating. Wherein the fiber Bragg grating (Fiber Bragg Grating, FBG) has been the most widely used in the real world, in fiber lasers, optical sensors, optical fiber has many applications in areas such as wavelength division multiplexing.

In 1978, Canada's Communications Research Centre developed the world's first optical fiber grating, Hill KO, who first discovered the fiber photosensitivity in germanium-doped silica fiber, such fiber will produce light induced grating effect, after use in Wave successfully fabricated a fiber grating periodicity. 10 years later, the United Technologies Research Center Meltz.G., Who invented a written ultraviolet-side optical fiber grating technology, the use of germanium-doped silica fiber to produce a Bragg resonance wavelength located within the communication band grating, which is the world's first. After, Hill and others have also invented a phase mask and 
written by point method, making practical fiber grating has been greatly improved, so that the production of reproducible fiber grating, suitable for large-scale production. Fiber Optic Sensors began to be used in transportation infrastructure, agriculture, geology health, military, aerospace, and so practical fields. Fiber grating began to commercialize practical. In practical terms, the end of the 1990s, Mendez Brown University in the United States and other people began to study the relevant fiber optic sensor for the detection of large civil structures of reinforced concrete, etc., after the United States, Britain and other Western countries, does not continue to invest The optical fiber sensing technology for health and safety monitoring dams, bridges, tunnels and other buildings. Nearly 10 years later, the United States is also a highway bridge installed multiple FBG sensors, this bridge with steel, the use of safety monitoring fiber grating structure. Over the same period, Germany also had the FBG strain sensor for monitoring tunnels, foundations and other static and dynamic strain, in many safety monitoring has been applied. During this period, the international community has been a lot of applied research FBG sensors for the construction of a variety of modern architecture and engineering of large-scale, practical methods have made great progress.

\section{FBG Sensor Fundamentals}

Fiber grating based photosensitive characteristics of the fiber material is made of. The so-called photosensitivity is doped fiber when UV irradiation is external, the inner fiber core refractive index caused by the interaction of a characteristic ion doping permanent change, the refractive index for divisions and ultraviolet light fiber core related to the spatial distribution of light intensity, the refractive index of the relative change in the size and change the size of the light intensity is linear, preserved to become the fiber grating. Fiber Bragg grating (FBG: fiber Bragg grating) is based on the principle of Mintel doped fiber ultraviolet light, use was spatially periodic intensity ultraviolet laser irradiation doped fiber, the fiber core is formed axially periodic refractive index distribution thereby forming a reflective optical fiber grating.

FBG is carried out by the outside world for its physical reflection center wavelength modulation sensing system to be used, when the FBG subjected to stress or temperature changes affect, FBG reflection center wavelength drift occurs, which directly reflects the wavelength accuracy or limit the entire precision sensing system. At the same time, the maturity of the demodulation technique is also directly related to the FBG sensing system to practical use process, because the current situation, the cost of high-resolution demodulation equipment are very high, occupies most of the cost of the entire sensor system , directly restricts the application of the promotion system, the study of fiber grating sensing signal demodulation techniques for is very important. Currently, there are a lot of signal demodulation method [27] to [31], there are spectral demodulation, matching fiber grating filtering, edge filtering, unbalanced scanning Michelson interferometer, tunable FP filter, tunable narrowband light source method.

\section{Settlement FBG Based Sensor Design and Packaging}

Sensor Design. The settlement sensing system uses a physical principle Unicom's works. Unicom vessel filled with water, to pass through Unicom pressure vessel of water. Pressure detection sensor is part of the settlement, which is based on the structure of FBG constituting the cantilever. Pressure changes in the system need to find a settlement to establish a reference point does not occur, the situation in other parts of the settlement are to do with this reference point, this time, you can feel the settlement sensor settlement reflects its installation point. In terms of the optical signal, settlement sensor measured pressure manifestations in the center wavelength of FBG changes, changes in the size of the theory is a linear relationship with the pressure on the system used to demodulate detected FBG center wavelength changes, you can We know the magnitude of the pressure, so in terms of a settlement of the case.

Settlement Sensor Package. Due to bare FBG diameter of only $125 \mu \mathrm{m}$, plus glass fiber inherently brittle break, characteristic susceptible to external mechanical damage in harsh environments practical application vulnerable to damage, making it difficult to use a single fiber 
grating in practice. Meanwhile, a simple naked of FBG in sensitivity is not high. In the package of research has also been carried out, we developed a variety of packaging materials and structures, greatly speeding up the practical, commercial pace of FBG. Although the FBG directly attached to the object to be measured is the easiest and most easy to implement the package, but this approach is not enough protection for FBG, vulnerable to environmental influences and external damage. So another simple way to develop is to FBG previously pasted on a substrate, which substrate is generally a metal sheet, and then the thus packaged sensors for installation, then the metal sheet may protect the FBG effect. However, this method still has some drawbacks, such as its sensitivity because the role of the metal sheet becomes low, while it is easily affected by the environment, such as humidity. To solve these problems, there have been a variety of packaging materials and packaging technology.

Sensor uses metal packaging, the sensing portion is mainly composed of two parts. Part 1 is the settlement of transfer means pressure level difference between the liquid and the diaphragm generated on the metal diaphragm, producing pressure $\mathrm{P}$, the diaphragm is deformed by the force, pushing the pressure transmitted to the cantilever thimble. Part 2 is composed by the Izod and adhesive surface on its FBG. Cantilever effective length $\mathrm{L}$ can be adjusted by changing the size of the housing base cantilever, thereby adjusting the sensitivity. The occurrence of a cantilever beam subjected to slight deformation thimble, this deformation acting on the FBG to produce axial strain, thus reflecting FBG center wavelength drift. When the sensor assembly, thimble first to give a pre-cantilever pressure to prevent the presence of free cantilever state and thimble.

Protective packaging is the most common, most basic kind of FBG sensor package. This form of packaging FBG sensors constitute sensors for different purposes other basic sensor element, the sensor settlement discussed in this paper, the basic cantilever sensor is based on this package so that FBG can be used to measure the pressure. The basic structure of this package is the use of glue or other bonding material will FBG tubular or sheet substrates together, such as a metal tube or sheet metal, used to measure the temperature or the stress of the outside world.

According to the working principle of FBG shows FBG strain and temperature of the cross-sensitivity characteristics. But in some practical applications, just to measure stress, such as this paper cantilever structure, the need to eliminate the influence of temperature. Commonly used methods to eliminate cross-sensitivity are dual wavelength matrix algorithms, double parameter matrix algorithms and temperature compensation method. Dual-wavelength method requires the use of two matrix operations FBG, let them together affected by temperature and strain through two simultaneous equations, and to compensate for the effects of temperature changes, this method requires the use of a multi-FBG, increase the mass additional costs sense system. Dual-parameter matrix calculation method requires the introduction of another strain and temperature at the same time sensitive and linear function, through this function and the simultaneous measurement of the actual function FBG by solving eliminate temperature effects.

Temperature compensation law is carried out by using a special substrate material for FBG package, such as the use of a material having a negative temperature coefficient, or the design of a feedback mechanism so that the effect of temperature is attached to the impact of stress up, played an offsetting the effect of temperature .

FBG temperature and stress sensitivity to meet the general applications, but in some special-purpose high sensitivity required, such as for packaging hydrophone, this time to take measures to improve the FBG temperature or pressure sensitivity. Sensitization principle is to use the package substrate material deformation led FBG strain together to improve the sensitivity of the sensor. For stress sensitization, a smaller Young's modulus can be used as a base material, then the base material is easily deformed, driving pasted thereon FBG strain together, thereby increasing the axial strain grating FBG of the stress sensitivity increase. Sensitization temperature thermal expansion coefficient is employed as the material of the substrate, so that the substrate temperature changes when the thermal expansion is more prone to deformation, it is attached to the FBG reflection center wavelength shift increases, thus increasing the temperature sensitivity. 


\section{Conclusion}

Currently, engineering structures and geological structure of the health and safety monitoring has great demand and foundation settlement monitoring has important practical significance in the building. This paper studies the FBG works and packaging technology. It puts forward multiplexing demodulation reference FBG present situation of the application, the propose cantilever and an FBG-based metal film structure for ground subsidence measurements. The design of the structure has been taken a lot of experimental research and analysis and makes a comprehensive understanding of the performance of the proposed structure and applies this structure to the actual settlement sensor cable tunnel structure subsidence monitoring system.

\section{References}

[1] Y. J. Rao, Z. L. Ran, R. R. Chen. Long-Distance Fiber Bragg Grating Sensor System with A High Optical Signal-to-Noise Ratio Based on A Tunable Fiber Ring Laser Configuration. Opt. Lett.,2006, 31(18):2684-2686.

[2] Morey W W. Meltz G. Weiss J M Recent Advances in Fiber Grating Sensors for Utility Industry Applications. 1997.

[3] Zeng-Ling Ran, Yun-Jiang Rao, Et Al. Miniature In-Line Photonic-Crystal-Fiber Etalon Sensorfabricated By 157nm Laser Micromachining. Optics Letters, 2007, 32(21): 3071-3073.

[4] Davis M. A, Kersey A. D. Matched Filter Interrogation Technique For Fiber Bragg Gratingarrays. Electron Lett. 1995, 31(10): 822-823. 